Coordinaciones regionales humanitarias. Exiliados, religiosos y organismos de derechos humanos en la formación de la Federación Latinoamericana de Asociaciones de Familiares de Detenidos Desaparecidos (1979-1982)

Mario Ayala

páginas / año 12 - n 29 Mayo-Agosto / ISSN 1851-992X/ 2020

http://revistapaginas.unr.edu.ar/index.php/RevPaginas

\title{
Coordinaciones regionales humanitarias. Exiliados, religiosos y organismos de derechos humanos en la formación de la Federación Latinoamericana de Asociaciones de Familiares de Detenidos Desaparecidos (1979-1982)
}

\author{
Regional humanitarian coordinations. Exiles, religious and human \\ rights organizations in the formation of the Latin American Federation \\ of Associations of Relatives of Disappeared Detainees (1979-1982)
}

\author{
Mario Ayala \\ Instituto de la Cultura, Sociedad y Estado; \\ Universidad Nacional de Tierra del Fuego; \\ Consejo Nacional de Investigaciones Científicas y Técnicas (Argentina) \\ mhayala@untdf.edu.ar
}

\begin{abstract}
Resumen
Este artículo analiza el rol de las redes transnacionales religiosas y de exiliados sudamericanos con base en Venezuela en la formación de la Federación Latinoamericana de Asociaciones de Familiares de Detenidos Desaparecidos (FEDEFAM). Lo hace a partir del análisis de las actividades de la Fundación Latinoamericana por los Derechos Humanos y el Desarrollo Social (FundaLatin), una organización ecuménica de derechos humanos fundada en 1978 en Caracas por iniciativa de un grupo de religiosos y exiliados chilenos y argentinos que trabajaban en la solidaridad con los refugiados y en la denuncia internacional de las dictaduras. La metodología escogida fue mayormente cualitativa, análisis crítico de documentos y realización de entrevistas orales. La hipótesis central es que las redes religiosas y de exiliados latinoamericanos con base en Venezuela fueron claves en la planificación, organización, coordinación y sostenimiento del proyecto de FEDEFAM entre el año 1979 y 1982, buscando una mayor articulación de las demandas y denuncias de las organizaciones nacionales de familiares en el ámbito regional e internacional.
\end{abstract}

Palabras clave

Redes transnacionales; derechos humanos; FundaLatin; exiliados; religiosos; FEDEFAM

\begin{abstract}
This article analyzes the role of transnational religious networks and South American exiles based in Venezuela in the formation of the Latin American Federation of Associations of Relatives of Disappeared Detainees (FEDEFAM). It does so from the analysis of the activities of the Latin American Foundation for Human Rights and Social Development (FundaLatin), an ecumenical human rights organization founded in 1978 in Caracas on the initiative of a group of religious and Chilean and Argentine exiles who worked in solidarity with refugees and in the international denunciation of dictatorships. The chosen methodology was mostly qualitative, critical analysis of documents, and oral interviews. The central hypothesis is that the Latin American religious and exile networks based in Venezuela were key in planning, organizing, coordinating and sustaining the FEDEFAM project between 1979 and
\end{abstract}

Esta obra está sujeta a la Licencia Reconocimiento-NoComercial-CompartirIgual 4.0 Internacional de Creative Commons. http://creativecommons.org/licenses/by-nc-sa/4.0/

(cc) BY-NC-SA 


\section{Mario Ayala}

1982, seeking greater articulation of the organizations' demands and complaints. nationals of relatives at regional and international level.

Keywords

Transnational networks; human rights; FundaLatin; religious; exiles; FEDEFAM

\section{Introducción}

Durante las confrontaciones ideológicas de la Guerra Fría y sus consecuentes violaciones masivas de los derechos humanos en la región (Roniger, 2018: 77), en particular durante las oleadas de represivas en el Cono Sur en la década del setenta, la denuncia y la solidaridad con las víctimas se estructuraron transnacionalmente en diversas redes integradas por actores que actuaban en el ámbito nacional, regional y global. Esta estrategia de trabajo en red más allá de las fronteras nacionales tuvo como objetivo unificador la defensa de los derechos humanos y la denuncia de sus violaciones en la esfera pública internacional para presionar a las dictaduras militares. Bajo la egida de la Doctrina de Seguridad Nacional (DSN) anticomunista estadounidense los regímenes militares de Argentina, Chile, Bolivia, Brasil, Uruguay y Paraguay aplicaron políticas represivas masivas y radicales como la tortura y la desaparición forzosa para desmovilizar a los sectores populares y lograr una reorganización socioeconómica y política interna (Pettiná, 2018: 136137).

De acuerdo a Keck y Sikkink (2000) este tipo de trabajo en redes puede definirse como una forma de organización en un patrón de acción no jerárquico y flexible entre actores e individuos entorno a un tema, con distinta intensidad de conexiones entre sus miembros y múltiples puntos de intersección con otros sujetos y redes. Las autoras conceptualizaron una red transnacional de defensa como

“... formada por actores que trabajan internacionalmente en torno a un tema, y que están vinculados por valores compartidos, por un discurso común y un denso intercambio de información y servicios. [...] El intercambio de información conforma el núcleo de la relación. La novedad de estas redes reside en la capacidad de los actores internacionales no tradicionales para movilizar información estratégicamente, con el objeto de ayudar a crear nuevos temas y categorías, y para persuadir, presionar e influir en organizaciones y gobiernos mucho más poderosos. En las redes, los activistas no sólo intentan influir en las consecuencias políticas, sino transformar los términos y la naturaleza del debate." (Keck y Sikkink, 1999:90).

Además, las redes trasnacionales de defensa se diferencian de otras redes por la posición central que ocupan en ellas los valores o principios en la motivación de su formación. Son significativas tanto a nivel transnacional, como regional y nacional y las que se centran en derechos humanos ponen recursos internacionales a disposición de nuevos actores de la política nacional y las luchas sociales (Keck y Sikkink, 1999:89-90). Con este marco analítico Keck y Sikkink estudiaron la eficacia 
Coordinaciones regionales humanitarias. Exiliados, religiosos y organismos de derechos humanos en la formación de la Federación Latinoamericana de Asociaciones de Familiares de Detenidos Desaparecidos (1979-1982)

de la red transnacional de defensa de los derechos humanos en América Latina de la década del setenta en relación al caso de Argentina bajo la última dictadura militar. Los golpes y la represión en países del Cono Sur de América Latina y Grecia habrían aumentado la conciencia global sobre las violaciones de los derechos humanos. Según las autoras, esta red transnacional de defensa integrada por ONG's creció en Europa y Estados Unidos y se complementó con las organizaciones derechos humanos que surgieron en toda América Latina para denunciar la represión de los gobiernos y brindar fuentes de información alternativa. A estos dos actores se agregó el trabajo internacional de derechos humanos que realizaron los exiliados (Keck y Sikkink, 2000:133). Sin embargo, su influyente investigación no abordó las redes regionales transnacionales de defensa de derechos humanos de la época, un tramo estratégico entre el nivel nacional y el internacional.

En este trabajo, siguiendo a Catoggio (2012; 2014; 2016), utilizamos la noción de redes religiosas transnacionales para hacer referencia a articulaciones flexibles de organizaciones confesionales (católicas y protestantes) y no confesionales de América Latina centradas en la protección de los derechos humanos y la asistencia a los represaliados, exiliados y refugiados en las décadas del setenta y ochenta. Estas redes brindaron sus relaciones, infraestructuras y recursos a las acciones de solidaridad y denuncia de las violaciones de los derechos humanos en la región. De acuerdo con la autora la literatura de los exilios latinoamericanos ha señalado la importancia del factor religioso en la conformación y desarrollo de redes de activismo trasnacional pero no cuenta con trabajos específicos que lo aborden (Catoggio, 2016: 187).

En cuanto a la noción de redes de exiliados (Roniger, 2011a; 2011b) el contexto internacional de la década del setenta brindó a los exiliados latinoamericanos la posibilidad de interactuar con redes transnacionales de activación política que les permitieron constituir estrategias de trabajo en red con otros exiliados y organizaciones del país de refugio alrededor de la defensa de los derechos humanos como una forma de lucha contra el régimen que los había excluido. Estas formas de articulación mejoraron sus oportunidades políticas y capacidades para efectuar acciones colectivas en los países de acogida y en la esfera pública internacional con el objetivo de generar cambios en el Estado expulsor (Roniger, 2011b: 42). En este contexto, como señala Roniger (2018: 29), la formación de organizaciones no gubernamentales junto con el activismo en las comunidades del exilio y las organizaciones civiles - y religiosas, agregamos nosotros- habrían de insertar el nuevo discurso de los derechos humanos bajo las dictaduras e impactar la evolución de normativa jurídica tras las transiciones a la democracia en América Latina.

Historiográficamente, el trabajo se inscribe entre dos campos de investigación de la historia contemporánea reciente de América Latina: los estudios sobre los exilios y la historia de las normativas, instituciones y movimientos sociales de derechos humanos, en particular aquellos que se desencadenaron frente a las dictaduras del 


\section{Mario Ayala}

Cono Sur (Alonso, 2008, 2010; Roniger, 2018; Sinkkink; 2018). Como han indicado Roniger (2018) y Sinkkink (2018), el aporte de los estados y los movimientos sociales latinoamericanos a la formulación de un discurso y un sistema internacional de derechos humanos tiene una larga historia que se remonta al siglo XIX. Para ambos, América Latina contribuyó al desarrollo de derechos e instituciones de derechos humanos con ideas, instituciones y normativas a nivel global. En el periodo reciente la región aportó al derecho internacional de derechos humanos con la figura legal de la desaparición forzada y los Juicios por la Verdad para conocer paradero de desaparecidos (Roniger, 2018: 77 y ss.). Estos dos estudios generales, también dan cuenta de una vacancia de investigaciones sobre organizaciones y redes regionales de derechos humanos con base en países latinoamericanos, que han comenzado a ser abordados en los últimos años (por ejemplo, en Catoggio, 2012, 2014, 2016; Ayala, 2014, 2017; Badan Ribeiro, 2016; Sagredo Mazuela, 2017).

El interrogante central que se intenta abordar en este artículo es el rol de las redes regionales transnacionales de religiosos y exiliados sudamericanos con base en Venezuela en la formación de la Federación Latinoamericana de Asociaciones de Familiares de Detenidos Desaparecidos (FEDEFAM). Lo hacemos a partir del análisis de las actividades de la Fundación Latinoamericana por los Derechos Humanos y el Desarrollo Social (FundaLatin), una organización ecuménica de derechos humanos fundada en 1978 en Caracas por iniciativa de un grupo religiosos y de exiliados chilenos y argentinos que trabajaban en la solidaridad con los refugiados y en la denuncia internacional de las dictaduras. La relevancia de este tema es clave para avanzar en el conocimiento empírico de las organizaciones regionales de derechos humanos con base en países del sur global durante las décadas de 1970/1980, y en la construcción de marcos teóricos-metodológicos adecuados para su estudio que den cuenta de su escala regional con una perspectiva transnacional y global, por el otro. La metodología escogida fue mayormente cualitativa, recurriendo al análisis crítico de documentos y la realización de entrevistas orales. Las fuentes de la investigación fueron las Actas de los congresos de la FEDEFAM de los años 1981 y 1982 (consultados en el Archivo de la organización en Caracas entre 2011 y 2012), entrevistas personales con exiliados que participaron de las actividades de FundaLatin y la FEDEFAM y los documentos que resguardaron en sus archivos personales.

La hipótesis que se propone es que las redes religiosas y de exiliados con base en Venezuela fueron claves en la planificación, organización, coordinación y sostenimiento del proyecto de FEDEFAM entre el año 1979 y 1982. Estos activistas religiosos y exiliados visualizaron como necesaria la articulación de iniciativas transnacionales para garantizar la coordinación regional de planes de acción, proyectos estratégicos y realización de encuentros sobre la política de desaparición forzada en la América Latina y el Caribe. Fue centralmente una forma de activismo transnacional que buscó una mayor coordinación de las demandas y denuncias de las organizaciones nacionales de familiares en el plano interno, regional e internacional. 
Coordinaciones regionales humanitarias. Exiliados, religiosos y organismos de derechos humanos en la formación de la Federación Latinoamericana de Asociaciones de Familiares de Detenidos Desaparecidos (1979-1982)

El artículo está organizado en cuatro partes y unas observaciones finales. En la primera parte se reconstruye el rol de FundaLatin en la organización del I Congreso Latinoamericano de Familiares de Detenidos Desaparecidos (Costa Rica, enero de 1981) que dio origen a la FEDEFAM. En la segunda se analiza el Plan de Acción de febrero-noviembre de 1981, definido por el I Congreso y llevado a adelante por el equipo coordinador de FundaLatin. La tercera se ocupa del II Congreso Latinoamericano de Familiares de Detenidos Desaparecidos que constituyó la FEDEFAM y de los actores y redes que colaboraron en él. La cuarta parte es dedicada a una panorámica del proyecto de FEDEFAM después de 1981.

FundaLatin y la organización del I Congreso Latinoamericano de Familiares de Detenidos Desaparecidos

FundaLatin fue fundada en Caracas en 1978 por un grupo de exiliados sudamericanos y venezolanos religiosos y laicos, como una organización ecuménica inspirada en la Teología de la Liberación (TL) y enfocada en la promoción integral de los derechos humanos en la región. Se constituyó con la visión estratégica que desde Venezuela se podía realizar un trabajo de solidaridad humanitaria hacia la región y el ámbito internacional, pues la existencia de libertades democráticas transformaban al país en un lugar seguro para realizar encuentros y gestiones públicas. Los proyectos principales de FundaLatin fueron la construcción de una red regional de denuncia de la situación de los derechos humanos y el impulso de federaciones regionales de organizaciones de derechos humanos. Las más exitosa de ellas fue la FEDEFAM, una organización regional centrada en la denuncia de las desapariciones forzadas como elemento común de las dictaduras militares y gobiernos autoritarios que se enmarcaban en la doctrina de Seguridad Nacional (FundaLatin, 1981a) anticomunista de la última etapa de la Guerra Fría (véase Ayala, 2014; 2017; Poveda Brito, 2011).

Las tareas organizativas iniciales para constituir la nueva organización regional estuvieron a cargo de un grupo de trabajo dirigido por el sacerdote Juan Vives Suria, ex presidente de Caritas venezolana y de la Comisión Católica de Migración, y director de FundaLatin, y Patrick Rice, un sacerdote obrero ex detenidodesaparecido en la Argentina, expulsado de este país a fines de 1976 y que partir de vivir esa experiencia límite decidió dedicarse a la lucha contra la política de desaparición forzada de personas. Tanto Vives Suria como Rice eran religiosos católicos en actividad seguidores de la Teología de la Liberación. Ambos tenían una vasta experiencia en el trabajo de solidaridad internacional con migrantes, exiliados y otras víctimas de violaciones de los derechos humanos, y formaban parte de redes religiosas y ecuménicas transnacionales. Por esta actividad conocían los mecanismos de denuncia internacional $y$ de gestiones ante organismos 


\section{Mario Ayala}

gubernamentales y multilaterales. Además, Rice, de origen irlandés, acreditaba una práctica previa en el trabajo humanitario en Estados Unidos e Inglaterra, entre 1977 y 1979, junto a otros religiosos, exiliados argentinos, y activistas locales, lo que lo convertía en un especialista en la materia al interior de FundaLatin (Entrevista con Patrick Rice, 12/12/2008, Ciudad de Buenos Aires). De modo que las trayectorias, experiencias de activismo trasnacional y relaciones anteriores de Vives Suria y Rice en el universo de actores religiosos de la región, así como el trabajo con exiliados y organizaciones internacionales de derechos humanos, les permitieron activar los contactos que facilitaron la coordinación del proyecto de Federación entre las organizaciones nacionales de familiares de desaparecidos, las redes religiosas regionales y las organizaciones de exiliados.

En esta tarea también fueron claves los capitales y relaciones de otros miembros del equipo fundador de FundaLatin, como Joaquín Undurraga, su vicepresidente, economista chileno exiliado en Caracas; Guido Zuleta, secretario general; Héctor Fagundes Ledesma, profesor de Derechos Humanos de la Universidad Central de Venezuela y la exiliada chilena Victoria Cáceres, entre otros (Entrevista con Patrick Rice, 12/12/2008, Ciudad de Buenos Aires). Todos ellos adscribían a la Teología de la Liberación, habían trabajado en la ayuda de los refugiados latinoamericanos en Venezuela y participado activamente de las campañas y disputas con los católicos conservadores para incidir en los debates de la III Conferencia General del Episcopado Latinoamericano que se realizó en Puebla, México, a inicios de 1979 (véase Jiménez Lozano, 1979). Los sectores de la Teología de la Liberación se oponían a las estructuras de injusticia y explotación en la región y a las dictaduras y las violaciones de los derechos humanos y con sus acciones consiguieron que la Conferencia concluyera ratificando el compromiso de los católicos latinoamericanos con la “opción preferencial por los pobres". Para Undurraga (1983) - promotor de la líneas estratégicas de FundaLatin, según Rice- la Teología de la Liberación llevaba implícita una visión de lo cristiano que, sin negar la doctrina, partía de los hechos de la realidad y permitía a los católicos el apoyo a las luchas de liberación, un dialogo con los marxistas y la construcción de alianzas con otros actores -como las organizaciones populares, los movimientos políticos y las iglesias protestantesentorno a la defensa de los derechos humanos en América Latina en un sentido integral.

De acuerdo a Patrick Rice, la idea de crear la FEDEFAM surgió en un encuentro entre activistas y familiares de Argentina, Uruguay, El Salvador, Chile y Bolivia, que concurrieron a la IX Reunión de la Asamblea General de la Organización de Estados Americanos (OEA), que tuvo lugar en La Paz (Bolivia) del 22 al 31 de octubre de 1979. Habían confluido allí para denunciar las violaciones de derecho humanos en sus países. La Central Obrera Boliviana (COB) habilitó sus instalaciones para alojarlos y facilitar sus reuniones. El día posterior al cierre de la reunión de la OEA, el 1 de noviembre, un golpe militar derrocó al presidente boliviano Walter Guevara Arze y la ciudad de La Paz fue ocupada por tropas militares que iniciaron una feroz represión sobre los sindicatos y toda oposición popular. La represión de esta 
Coordinaciones regionales humanitarias. Exiliados, religiosos y organismos de derechos humanos en la formación de la Federación Latinoamericana de Asociaciones de Familiares de Detenidos Desaparecidos (1979-1982)

dictadura de catorce días encabezada por el coronel Natusch Busch pasó a la historia como la masacre de Todos Santos que, de acuerdo a diversas fuentes, provocó de 100 a 200 muertos y 40 a 125 desaparecidos (véase Dunkerley, 2003; Mealla, 2013). En aquel contexto boliviano, y a nivel regional con la visita de la Comisión Interamericana de Derechos Humanos de la OEA (CIDH) a Argentina en septiembre, recordó Rice, los activistas de derechos humanos y los familiares se convencieron de la necesidad de "profundizar los vínculos de unión a través de una reunión más formal" para denunciar la política de desapariciones forzadas de la dictaduras de la región. La idea comenzó a concretarse un año más tarde, cuando FundaLatin, en coordinación con la Comisión Ecuménica Pro Derechos Humanos de Costa Rica (CEPRODHU), tuvo la iniciativa de constituir un grupo de trabajo para organizar el Primer Congreso Latinoamericano de Familiares de Desaparecidos, que sesionó en un convento de San José, Costa Rica, entre el 20 y el 23 de enero de 1981 (Entrevistas con Patrick Rice, 12/12/2008 y 10/03/2010, Ciudad de Buenos Aires; Rice, 2009).

De acuerdo con los documentos consultados y testimonios reunidos, las razones para organizar la FEDEFAM como una estrategia de coordinación regional de organizaciones de derechos humanos centrada en la denuncia de la desapariciones forzadas, fueron la baja coordinación de las organizaciones nacionales y su necesidad de acceder a las organizaciones no gubernamentales y organismos internacionales de derechos humanos para realizar las denuncias, ante un escenario de regionalización de la práctica represiva y la coordinación de las dictaduras y gobiernos autoritarios para eliminar opositores. La centralidad de este tema para los organizadores fue expuesta en el Documento Base de FundaLatin en el I Congreso de Familiares Desaparecidos de América Latina, con el que se iniciaron las discusiones:

\footnotetext{
"En los golpes militares que, en la década del 70, se suceden en Uruguay, Bolivia, Chile, Argentina, Perú, Centro-América y nuevamente en Bolivia, se constata una progresiva y eficiente coordinación de los aparatos represivos de las dictaduras, implementando y extendiendo el método de las desapariciones, en una muestra más de su desprecio por derechos fundamentales del hombre.

[...] La falta de coordinación de los organismos [de familiares de víctimas de desaparición forzada] de distintos países y la constante represión y legislación que, en nombre de la Seguridad Nacional, se dan los factores que detentan el poder en cada país. Las relaciones entre los organismos son esporádicas, coyunturales y en algunos casos, muy pocas, productos de una serie de factores que se quiere profundizar y aportar en este congreso, lográndose alguna forma de respuesta coordinada ante los planes monolíticos y perfectamente coordinados del enemigo." (FundaLatin, 1981무)
}

Ahora bien ¿Por qué las denuncias y demandas por las desapariciones forzadas fueron planteadas en términos de derechos humanos y parentesco? Para responder a este interrogante, siguiendo a Elizabeth Jelin, no debe pasarse por alto la 


\section{Mario Ayala}

centralidad que tuvieron los lazos de familia en la construcción de la figura del desaparecido como víctima, y en la búsqueda de verdad y justicia, en los contextos políticos dictatoriales de represión y censura donde las actividades de organizaciones políticas y sindicatos estaban suspendidas (Jelin en Crenzel, 2010). Los miembros de FundaLatin visualizaron el poder de este reclamo cuando era realizado por sus familiares y decidieron darle un apoyo de coordinación regional y proyección internacional.

En los recuerdos de dos organizadores del I Congreso, Patrick Rice por FundaLatin y Viviana por Amnistía Internacional-sección Venezuela (Entrevistas con Patrick Rice, 12/12/2008 y 10/03/2010, Ciudad de Buenos Aires; Entrevista con Viviana, 06/12/2008, City Bell, La Plata), hallamos elementos importantes que permiten acceder a los motivos que los llevaron a impulsar el proyecto y a sus percepciones del momento. Estos relatos enfatizaron en primer lugar la sensación de desconcierto e incertidumbre que los atravesaba frente a la masividad y regionalización de las desapariciones forzadas. En segundo lugar, destacaron las condiciones locales en que se realizó el evento. La escasa colaboración del gobierno de Costa Rica, por ejemplo otorgando visas de cortesía de pocos días a los participantes, o negándose a brindar seguridad al Convento donde se realizó el evento, "a pesar de que había un gran temor por la seguridad y se temía que las dictaduras actuaran en forma conjunta y realizaran secuestros masivos" y/o que se infiltren sus servicios de inteligencia (Correspondencia con Viviana, 21/07/2019, Córdoba). Además, el I Congreso recibió la cobertura periodística de Radio Noticias del Continente(RNC) , emisora de onda corta con una potente señal de alcance continental creada a mediados de 1979 con recursos humanos y financieros de la organización políticomilitar argentina Montoneros, que transmitió en directo algunas de sus partes (Entrevistas con Patrick Rice, 10/03/2010, Ciudad de Buenos Aires).

A pesar de todos estos condicionantes el I Congreso de Familiares Desaparecidos de América Latina se realizó y contó con una importante participación que puso en evidencia la amplitud de la red transnacional de defensa de derechos humanos que en aquel momento articulaba FundaLatin a nivel regional y mundial; y que incluía un extenso espectro de relaciones y apoyos que le permitieron organizar el congreso en un tercer país y garantizar la colaboración de diversos actores claves. En primer lugar, se observó la participación de delegaciones de asociaciones y organizaciones integradas por familiares de desaparecidos de ocho países, con residencia en países de origen, en el exilio y/o en terceros estados, a saber: Argentina, Chile , El Salvador, Guatemala, Haití, México , Paraguay y Uruguay (FEDEFAM, 1981ạ: 274-276).

Segundo, el Congreso contó con un fuerte respaldo de organizaciones de derechos humanos, religiosas, de exiliados y de solidaridad con base en países de origen y en el exterior. Las organizaciones dedicadas al caso de Argentina fueron Servicio de Auxilio Católico (Argentina), Grupo Defensa de los Derechos Humanos en Argentina (Canadá), Comité de Solidaridad con el Pueblo Argentino de New York, Comité Argentino de Solidaridad y Revista Denuncia (Estados Unidos), Comité Argentino de Defensa de Derechos Humanos (CADHU), Trabajadores Argentinos de la Salud 
Coordinaciones regionales humanitarias. Exiliados, religiosos y organismos de derechos humanos en la formación de la Federación Latinoamericana de Asociaciones de Familiares de Detenidos Desaparecidos (1979-1982)

Mental, Unidad y Resistencia Argentina en el Exilio (URAE), Comunidad de Cristianos Argentinos (México). Las que hacían foco en Bolivia, Comité Costarricense de Solidaridad con Bolivia y Comité de Solidaridad con la A.P.D.H en Bolivia en Lima. Las dedicadas a Chile el Comité de Solidaridad con Chile (Costa Rica) y el Instituto de Estudios Políticos-Proyecto Derechos Humanos (Estados Unidos). Para Guatemala, Comité Ecuménico Pro Derechos Humanos (Guatemala), Comité Costarricense de Solidaridad con Guatemala (Costa Rica) Comité Internacional por la vida de Alaide Foppa (España y México). Y para el caso de Paraguay se hizo presente el Comité de Iglesias de Paraguay. La delegación de organizaciones e instituciones locales estuvo integrada por el Consejo Superior Universitario Centroamericano, Comité Ecuménico Pro Derechos Humanos, Partido Socialista Costarricense, Juventud Democrática Cristiana, CENAP, CSUCA-Programa de Solidaridad (FEDEFAM, 1981aa: 274-276). Mientras que la delegación venezolana quedó compuesta por miembros de la comisión organizadora de FundaLatin, representantes de la sección venezolana de Amnistía Internacional y de la Cruz Roja y miembros de la Coordinadora Pro Derechos Humanos en la Argentina (CPDHA), un grupo de acción solidaria formado por exiliados argentinos en Caracas hacia fines de 1980 para apoyar a los organismos de derechos humanos del interior de Argentina y denunciar a la dictadura militar (Véase Ayala, 2017).

En tercer lugar, participó un nutrido grupo de observadores y representantes de los gobiernos de Costa Rica, Venezuela, Nicaragua; de la oficina regional del Alto Comisionado de las Naciones Unidas para los Refugiados (ACNUR) ; y personalidades y organismos de solidaridad y derechos humanos de América Latina, Europa y Estados Unidos .

Las conclusiones del I Congreso se publicaron en una Declaración con cuatro definiciones cardinales. Primero, realizar una convocatoria a todos los organismos de familiares de desaparecidos del continente (en países bajo represión, en el exilio o en terceros países) a crear una conciencia continental sobre la aberrante práctica represiva "de hacer desaparecer al adversario". Segundo, buscar un mayor intercambio y coordinación entre las organizaciones nacionales de familiares de desaparecidos para aumentar la eficacia de sus reclamos. Tercero, proponer a las organizaciones internacionales como la Organización de las Naciones Unidas (ONU) y la Organización de los Estados Americanos (OEA) "desarrollar un nuevo ordenamiento jurídico" que permitiera encuadrar la novedad de la forma represiva de las desapariciones forzadas de personas. Y cuarto, trabajar de forma coordinada a nivel internacional para denunciar la práctica de las desapariciones forzadas, la coordinación represiva y el tráfico internacional de prisioneros de las dictaduras militares de la región (Equipo de Coordinación FundaLatin, 1981b; Revista 2001, 1981:11)

A nivel organizativo y de plan de acción, el Congreso tomó cinco decisiones y delegó su ejecución en el grupo coordinador de FundaLatin, a saber: 1) la organización de 


\section{Mario Ayala}

un segundo Congreso en Caracas a fines de 1981 para la creación de una "Federación de Familiares de Detenidos Desparecidos de América Latina”; 2) el nombramiento de Patrick Rice como coordinador ejecutivo del proyecto; 3) la realización de una campaña internacional entre el 25 y 31 mayo de 1981 bajo el lema la "Semana del detenido desaparecido"; 4) la constitución de un grupo de trabajo de juristas con el objetivo de elaborar un proyecto de convención contra la desaparición forzosa e involuntaria de personas que la declarara como un crimen de lesa humanidad; y 5) la realización de una gira por la región encabezada por Rice para contactar e informar sobre el proyecto de la Federación.(Equipo de Coordinación FundaLatin, 1981a, 1981b) En cumplimiento del mandato los miembros de FundaLatin regresaron a Venezuela y desde allí comenzaron a trabajar, activando y ampliando sus relaciones y redes en busca de apoyos y asesoramiento con el objetivo de cumplir con el mandato organizativo y el Plan de Acción acordado para 1981.

\section{El Plan de Acción de febrero-noviembre de 1981}

La primera actividad del grupo coordinador de FundaLatin fue la organización y lanzamiento de la campaña internacional de la "Semana del Detenido Desaparecido" entre el 24 y el 31 de mayo de 1981. La campaña, una actividad de alcance transnacional, fue coordinada con organizaciones de familiares de desaparecidos (en el interior y en el exilio), organizaciones de exiliados y de solidaridad de América Latina, Europa y los Estados Unidos. Inspirada en las campañas de Amnistía Internacional, se concentró en informar y denunciar sobre la práctica específica de la desaparición forzada de personas con la intención de influir en la opinión pública nacional e internacional, los gobiernos, las iglesias y los organismos intergubernamentales. A partir de estas acciones se esperaba conseguir apoyos públicos y gubernamentales a fin de concretar la promulgación de una Convención Internacional contra las Desapariciones Forzadas (Equipo de coordinación FundaLatin, 1981aㅜ; Entrevista con Patrick Rice, 12/12/2008, Ciudad de Buenos Aires).

La segunda actividad del Plan de Acción fue convocar a las asociaciones de familiares de desaparecidos de países que no habían participado de la reunión de Costa Rica. En esta línea, Rice como coordinador ejecutivo del proyecto de Federación realizó una gira por Brasil en junio de 1981 intentando contactar y sumar a organizaciones de este país. La gira fue positiva, en tanto se sumaron al proyecto el Comité por la Amnistía en el Brasil y el Comité Brasilero de Solidaridad con los Pueblos de América. Asimismo, se planteó el tema de la inclusión en la Federación de los familiares de desaparecidos en terceros países (por ejemplo, brasileros, chilenos, paraguayos y uruguayos desaparecidos en la Argentina) junto a las asociaciones locales (Equipo de Coordinación FundaLatin, 1981aa 1981b).

La tercera actividad del equipo de coordinación fue gestionar la constitución de un grupo de juristas que elaborara un proyecto de Convención contra las 
Coordinaciones regionales humanitarias. Exiliados, religiosos y organismos de derechos humanos en la formación de la Federación Latinoamericana de Asociaciones de Familiares de Detenidos Desaparecidos (1979-1982)

Desapariciones Forzadas y buscar manifestaciones de apoyo parlamentario a la misma. Para cumplir con el primer objetivo recurrieron a agrupaciones de juristas en el exilio como la Comisión Argentina de Derechos Humanos (CADHU) , a Amnistía Internacional (AI), y a profesionales de renombre internacional (véase por ejemplo: Patrick Rice al jurista Leonte Herdocia 27/07/1981, en FEDEFAM, 1981ạ: 329-330). Mientras que para el segundo activaron sus contactos políticos en Venezuela y en mayo de 1981 lograron una declaración del Congreso de este país a favor de la creación de una Convención Internacional contra las Desapariciones Forzadas.

La cuarta línea de acción del grupo coordinador fue intentar apoyar con información y acciones al Grupo de Trabajo sobre Desapariciones Forzadas de la Comisión de Derechos Humanos de la ONU (GTDFONU), creado el 29 de febrero de 1980 por la Comisión de Derechos Humanos del organismo con un año de mandato . Las acciones de los activistas demandaban la ampliación del mandato del GTDFONU y buscaban en simultáneo garantizar la articulación de las organizaciones de familiares con él. Un paso importante de la estrategia era buscar que la futura FEDEFAM se constituyera como una organización no gubernamental con estatus consultivo ante la ONU (Entrevistas con Patrick Rice, 12/12/2008 y 10/03/2010, Ciudad de Buenos Aires).

La quinta línea de acción que ocupó al equipo dirigido por Rice fue la realización de gestiones a nivel nacional e internacional para garantizar la logística y funcionamiento del II Congreso de Familiares de Detenidos de América Latina programado para fines de noviembre de 1981 en Caracas, tema que se abordará ampliamente en el apartado siguiente.

En simultáneo con estas gestiones para garantizar del Plan de Acción de 1981, los coordinadores del proyecto de Federación se interesaron por la búsqueda modelos organizativos y métodos de protesta no violentos como campañas y acciones comunicativas orientadas a la opinión pública de países democráticos y desde estas a la esfera pública internacional. Estas experiencias fueron un importante insumo para la organización de la semana mundial de los detenidos-desaparecidos, que combinó las innovaciones de las organizaciones locales de familiares como las Madres de Plaza de Mayo (véase el respecto Jelin en Crenzel 2010) y de ONGs internacionales como Amnistía Internacional (AI). A la vez buscaron intercambios de experiencias con organizaciones transnacionales ecuménicas y de derechos humanos, estudiando sus modelos organizativos y repertorios de acción, tanto para FundaLatin como para la naciente FEDEFAM (véase, por ejemplo: Patrick Rice a Gianni Novello de Pax Christi Internacional, 27/07/1981, en FEDEFAM, 1981ạ: 300). La documentación consultada permite destacar que además de la búsqueda de articulaciones, apoyos e intercambios, el equipo de FundaLatin se interesó por la búsqueda de métodos de lucha no violentos y fundamentados en los principios de los derechos humanos, el ecumenismo cristiano y los valores humanistas. 


\section{Mario Ayala}

\section{El II Congreso de Familiares de Desaparecidos de América Latina y la constitución de FEDEFAM}

Después de diez meses de gestiones y actividades de visualización de la política de desapariciones forzadas en los ámbitos nacionales e internacionales, el grupo organizador de FundaLatin garantizó la realización del II Congreso de Familiares de Detenidos-Desaparecidos de América Latina en Caracas, los días 24 y 28 noviembre de 1981. Bajo el lema "Hasta Encontrarlos", el evento tuvo como objetivo central constituir la FEDEFAM, definiéndola como un "mecanismo de denuncia de esta gravísima violación a nivel mundial y de protección y coordinación de los familiares" que permitiera "formular un programa de actividades" y "propiciar el estudio y la adopción de una Convención Internacional que ayudará a erradicar las detenciones y desapariciones" (FEDEFAM, 1981b: 1). Por este motivo, las sesiones del II Congreso discutieron intensamente el establecimiento de los estatutos de la Federación que definieron sus objetivos, métodos de acción, modalidades de funcionamiento, características de sus miembros y la estructura y formas de elección de autoridades (FEDEFAM, 1981b). A su vez, el encuentro ratificó la continuidad de Patrick Rice como su Secretario Ejecutivo y creó su boletín informativo bimestral Hasta Encontrarlos, que comenzó a publicarse en mayo de 1982 (FEDEFAM, 1982ª). El estudio de la documentación del II Congreso y las memorias de los activistas que lo organizaron revelan que la convocatoria logró reunir a 130 delegados de los actores meta del proyecto. La lista de estos actores incluyó asociaciones de familiares de desaparecidos y presos políticos, comités de exiliados, comités de solidaridad y organizaciones derechos humanos con base en América Latina, Europa, Estados Unidos y Canadá. El análisis de la información de estas organizaciones por país de origen y de residencia nos permitirá plantear dos cuestiones centrales para este trabajo: la amplitud de la red regional de derechos humanos construida por los religiosos y exiliados miembros de la red de FundaLatin, por un lado; y por otro, dimensionar el trabajo de las redes de exiliados del Cono Sur en ella, en particular las de argentinos. Veamos cómo estas dos cuestiones se presentaron en las fuentes estudiadas.

Primeramente, observamos un elevado número de formaciones con foco en países del Cono Sur. Las organizaciones orientadas al caso de Argentina fueron las más numerosas constituyendo un total de catorce, entre las cuales se contaban Asociación Madres de Plaza de Mayo, Asociación Abuelas de Plaza de Mayo, Comisión de Familiares de Desaparecidos y Detenidos por Razones Políticas (las tres operando dentro del país), delegaciones de la COSOFAM con sede en Holanda, Barcelona, Italia, México, CADHU (México) y agrupaciones de exiliados de México (Unidad y Resistencia Argentina en el Exilio, Trabajadores Argentinos de la Salud Mental y Comunidad Cristiana Argentina), Venezuela (Coordinadora Pro Derechos Humanos en la Argentina, Grupo de Solidaridad de Ciudad Bolívar) e Italia (Comité Antifascista contra la Represión en Argentina). Le seguían seis organizaciones con interés en Chile: Agrupación de Familiares de Detenidos-Desaparecidos de Chile, 
Coordinaciones regionales humanitarias. Exiliados, religiosos y organismos de derechos humanos en la formación de la Federación Latinoamericana de Asociaciones de Familiares de Detenidos Desaparecidos (1979-1982)

Agrupación de Familiares de exiliados de Concepción, Comisión de Derechos Humanos (las tres operando en el interior del país), Agrupación de Familiares de Detenidos en Chile (México), Comité de Apoyo a los Presos Políticos y Familiares de Detenidos-Desaparecidos de Chile (Venezuela) y el Instituto de Estudios PolíticosProyecto Derechos Humanos (Estados Unidos). Para el caso de Paraguay fueron cuatro: Comisión de Derechos Humanos (Paraguay), Agrupación de Familiares de Paraguayos Desaparecidos (Argentina) y el Comité de Solidaridad por Detenidos Desaparecidos en Paraguay y la Asociación Cultural Democrática de Paraguay (Venezuela). Mientras que otras cuatro apuntaban a Uruguay: Madres del Uruguay con Familiares de Detenidos Desaparecidos en la Argentina (Uruguay), Colectivo por la Defensa de los Rehenes de Uruguay (Venezuela), Asociación de Familiares de Desaparecidos Uruguayos (México), Agrupación de Familiares de Uruguayos Desaparecidos (Francia).

En contraste, las organizaciones centradas en países de Centroamérica, México y el Caribe constituyeron un pequeño grupo. Para el caso de El Salvador participaron el Comité de Madres y Familiares de Desaparecidos, Reos Políticos y Asesinados y la Comisión de Derechos Humanos de El Salvador, que operaban dentro del país. Para Guatemala, la Comisión de Derechos Humanos en Centro América y el Comité de UNAM de Guatemala, ambos con sede en Costa Rica. Para el caso de Haití, el Comité Haitiano de Solidaridad (Venezuela) y el Comité para la Liberación de Sylvio Claude y demás Presos Políticos de Haití (Estados Unidos). Y las organizaciones con foco en México: el Comité Pro-Defensa de Presos, Perseguidos, Exiliados y Desaparecidos Políticos de México y el Frente Nacional contra la Represión (FEDEFAM, 1981b: 13).

En segundo lugar, el II Congreso consiguió la presencia de 29 observadores de organizaciones gubernamentales, no gubernamentales y religiosas nacionales e internacionales, que dieron un respaldo clave a la iniciativa y la visualizaron a nivel local, regional e internacional. Consideramos importante enumerar a estas organizaciones en el orden que lo hicieron los organizadores: Grupo de Trabajo sobre Desapariciones Forzadas e Involuntarias (Naciones Unidas), Comisión Internacional de Juristas, Amnesty International (Inglaterra), Amnesty International (Alemania), Amnesty International (Estados Unidos), Amnesty International (Venezuela), Paz y Desarrollo (Canadá), Derechos Humanos Internet (sic) (Estados Unidos), Fundación Monseñor Romero (Bélgica), Fiscalía General de la Republica (Venezuela), Servicio Social Internacional (Venezuela), Equipo Eclesial Coro de Venezuela, Hermanas Carmelitas de Venezuela, Hermanas de San José de Tarbes de Venezuela, Universidad Católica Andrés Bello (Venezuela), Comité de Apoyo (Los Teques), Embajada de Holanda en Venezuela, Frente Amplio de Uruguay (Venezuela), C.N.T. del Uruguay (Venezuela), Partido Demócrata Cristiano (Haití), Comité de Apoyo a los Presos Políticos-El Hatillo (Venezuela), Equipo Eclesial Nueva Tacagua (Venezuela), Comisión Justicia y Paz de Catia (Venezuela), Grupo Petare 


\section{Mario Ayala}

(Venezuela), Fundación La Sallé de Ciencias Naturales (Venezuela), Universidad de los Andes (Venezuela), Centro Pellín (Caracas), ITER (Caracas), CEVEJ (Caracas) (FEDEFAM, 1981b: 1-2).

Este listado de organizaciones e instituciones observadoras del Congreso nos permite conocer la densidad de la red humanitaria articulada por FundaLatin y las redes de exiliados en esta coyuntura. En primer término destaca como significativa la presencia del GTDFONU, la Comisión Internacional de Juristas, secciones de AI, la Fiscalía General de la República de Venezuela, Derechos Humanos Internet, Fundación Monseñor Romero y el Servicio Social Internacional; y en segundo término, una importante participación de actores locales, principalmente de redes religiosas y ecuménicas -incluidas organizaciones eclesiales de la zona de Caracas y alrededores-, secundadas por dos universidades.

En tercer lugar, el II Congreso recibió 130 mensajes y adhesiones de religiosos, organizaciones de derechos humanos, sindicatos, partidos, embajadas, comités, universidades y autoridades de gobierno de 29 países de América y Europa .

Esta evidencia nos permite hacer las siguientes observaciones. En primer lugar, indica que la práctica de la desaparición forzada era motivo de preocupación o de resistencia en toda la región. En segundo lugar, muestra el peso determinante de las organizaciones de derechos humanos (tanto en el interior como en el exilio), redes de religiosos y exiliados con foco en países del Cono Sur: Argentina (14), Chile (6), Paraguay (4) y Uruguay (4). En tercer lugar, señalan la importancia de las organizaciones de solidaridad y defensa de los derechos humanos integrados por exiliados latinoamericanos con base de acción en Venezuela (8), México (6), España (2) -principales destinos de la ola de exiliados de los setenta- y resto de Europa (4). Por último, señala que las organizaciones centradas en Argentina que se encontraban en el exterior sumaban un total de 10 y sólo 4 tenían residencia en su país, mientras que para Chile esta relación era de $3 / 3$, y para Uruguay y Paraguay de 3/1. Lo cual señala el peso que tuvo en la creación de FEDEFAM el activismo de las organizaciones de exiliados y agrupaciones de familiares de desaparecidos que operaban desde el exilio.

En suma, el análisis de las organizaciones observadoras, adherentes y participantes II Congreso de Familiares de Desaparecidos de América Latina, nos permite destacar rol determinante de las redes transnacionales religiosas y de exiliados en la articulación en una estrategia regional y transnacional de organizaciones de familiares de desaparecidos que fue FEDEFAM. En el caso de la red religiosa humanitaria regional de la que formaba parte FundaLatin, su contribución fue clave en la concepción, gestión y realización del proyecto de la FEDEFAM. Como ha señalado Soledad Catoggio, la trama religiosa de las redes humanitarias y el activismo transnacional durante las dictaduras del Cono Sur fueron claves "en la gestión de la circulación y los intercambios entre las víctimas dentro y fuera del país; los dotó de una infraestructura y de una serie de recursos que fueron claves para la asistencia a las víctimas y sus familiares y para la denuncia de las dictaduras" (Catoggio, 2014: 187). Respecto de las redes de exiliados del Cono Sur, su 
Coordinaciones regionales humanitarias. Exiliados, religiosos y organismos de derechos humanos en la formación de la Federación Latinoamericana de Asociaciones de Familiares de Detenidos Desaparecidos (1979-1982)

participación en el proyecto de Federación debe entenderse como parte de una estrategia colectiva de trabajo político en redes amplias, tanto en el país de refugio como a nivel regional-transnacional, con el objetivo de potenciar la denuncia de las violaciones a los derechos humanos y el apoyo a las organizaciones que trabajaban en el interior del país (Ayala, 2014; 2017).

FundaLatin y el proyecto de FEDEFAM después de 1981

La documentación de la FEDEFAM muestra que las redes transnacionales religiosas y de exiliados fueron claves en la organización, coordinación y sostenimiento del proyecto de la FEDEFAM hasta el año 1982. Si bien en el II Congreso quedó constituida la FEDEFAM, como una organización de denuncia de las desapariciones forzadas y de protección y coordinación de las organizaciones de familiares, se decidió delegar nuevamente en FundaLatin la organización de su III Congreso y la coordinación del trabajo en el proyecto de una Convención Internacional para la Protección contra las desapariciones forzadas que debía presentarse en el mismo (FEDEFAM, 1981b: 2). Sobre la base de ese mandato FundaLatin organizó el III Congreso Latinoamericano de Familiares de Detenidos Desaparecidos en Lima, Perú, del 4 al 8 de noviembre de 1982, con la ayuda de su red de organizaciones religiosas y ecuménicas defensoras de derechos humanos, entre las cuales la más cercana en nivel local era la Comisión Evangélica Latinoamericana de Educación Cristiana (CELADEC) (Entrevista con Patrick Rice, 12/12/2008, Ciudad de Buenos Aires). El encuentro funcionó en dependencias gestionadas por el CELADEC, logró una importante repercusión mediática y política local-regional, y cumplió con el objetivo central de consensuar un proyecto de Convención contra la Desaparición Forzada de Personas -que fue remitida a Estados miembros de Naciones Unidas y otras instancias internacionales - y en la constitución de las autoridades de la Federación mediante una dirección colegiada (véase FEDEFAM, 1982).

A partir de este III Congreso, FEDEFAM inició un lento proceso de consolidación que la llevó a autonomizarse de FundaLatin, estableciendo una sede propia en Caracas hasta ese momento funcionaba en las oficinas de FundaLatin-desde donde realizaron el trabajo de coordinación y archivo. Sin embargo -de acuerdo al testimonio de Rice- más allá de las declaraciones, resoluciones y acuerdos de los congresos, FEDEFAM tuvo poca capacidad para llevar adelante las campañas definidas en sus planes de acción debido a su condición de organización de segundo grado subordinada a las dinámicas de las organizaciones nacionales y a los contextos represivos en que actuaban. De modo que la Dirección y Secretaría Ejecutiva de la FEDEFAM en ningún período contaron con autonomía relativa para llevar a cabo acciones y posicionamientos que no hubieran sido acordados y discutidos por cada 


\section{Mario Ayala}

organización integrante. La mayoría de la cuales, además, actuaban en un contexto de inseguridad, represión y hostigamiento y en una situación de emergencia continua. Por estas razones su trabajo se concentró mayormente en tres actividades: 1) gestiones ante organismos internacionales y Estados para lograr la aprobación del proyecto de Convención Internacional; 2) coordinación de la semana internacional del detenido-desaparecido; y 3) organización de los congresos (Entrevistas con Patrick Rice, 12/12/2008 y 10/03/2010, Ciudad de Buenos Aires). Sin embargo, en las dos décadas siguientes FEDEFAM permitió a las organizaciones de afectados de la región potenciar la denuncia contra sus Estados en el ámbito internacional y, como resultado, lograr incrementar las presiones externas sobre ellos para que modificaran su política de violaciones a los derechos humanos. En Venezuela en este trabajo tuvieron un desempeño importante la red religiosaecuménica de FundaLatin y la red de exiliados latinoamericanos en este país, en especial los sudamericanos y entre estos los argentinos que tenían residencia en Caracas y ciudades cercanas (Ayala, 2017).

Con el inicio de la transición a la democracia en el Cono Sur a mediados de los ochenta muchos exiliados retornaron a sus países, pero FEDEFAM continuó trabajando en la lucha internacional contra la política de desapariciones forzadas. Actualmente es reconocida como la ONG latinoamericana que tuvo un rol central en las campañas internacionales que contribuyeron a la adopción por la Asamblea General de ONU de la Declaración sobre la protección de todas las personas contra las desapariciones forzadas, en 1992, y posteriormente a la sanción de la Convención Contra las Desapariciones Forzadas por la OEA (1994) y las Naciones Unidas (aprobada en diciembre de 2006 y ratificada y efectivizada en diciembre de 2010).

\section{Observaciones finales}

Después de confrontar los objetivos de esta investigación con las fuentes y reflexionar sobre los resultados, nuestra conclusión principal es las redes religiosas y de exiliados sudamericanos con base en Venezuela fueron claves en la planificación, organización, coordinación y sostenimiento del proyecto de FEDEFAM entre el año 1979 y 1982.

La trayectorias y experiencias previas de los sacerdotes Vives Suria, Patrick Rice y el equipo fundador de FundaLatin, y su relación en las redes del exilio conosureño y latinoamericano les permitieron activar los contactos y redes internacionales que facilitaron la coordinación del proyecto de la FEDEFAM. A su vez, el estudio de las redes de los exiliados sudamericanos que participaron de la formación de la FEDEFAM demuestra la existencia de dinámicas redes transnacionales de lucha contra las dictaduras militares centradas en la denuncia de las violaciones de los derechos humanos, que articularon a las agrupaciones de solidaridad y derechos humanos formadas en el exilio con las organizaciones que realizaban el mismo trabajo en el interior del país. 
Coordinaciones regionales humanitarias. Exiliados, religiosos y organismos de derechos humanos en la formación de la Federación Latinoamericana de Asociaciones de Familiares de Detenidos Desaparecidos (1979-1982)

Puede afirmarse que los religiosos y exiliados chilenos y argentinos miembros de FundaLatin y sus redes regionales visualizaron el poder de los lazos de familia en la construcción de la figura del desaparecido como víctima; y la necesidad de articulación de iniciativas transnacionales para garantizar la coordinación regional de organizaciones de familiares nacionales en planes de acción, proyectos estratégicos y realización de encuentros contra la política de desaparición forzada en la América Latina. Para lograr estos objetivos desarrollaron una forma de activismo transnacional que impulsó y apoyó la formación de una organización regional de organizaciones de familiares de desaparecidos. Mejorando la protección de las organizaciones en el terreno y permitiéndoles coordinar actividades de denuncia internacional en colaboración otras redes transnacionales de defensa de derechos humanos y al mismo tiempo impulsar la adopción de una Convención Internacional contra las desapariciones forzadas.

En definitiva, la red transnacional de defensa que dio origen a FEDEFAM no solamente se ocupó de coordinar a las organizaciones nacionales a nivel regional y mejorar las acciones de denuncia internacional. Si no que buscó presionar e influir en gobiernos $\mathrm{y}$ organismos intergubernamentales para crear nuevos temas $\mathrm{y}$ categorías que apuntaban a transformar los términos y la naturaleza del debate: considerar a la práctica represiva de la desaparición forzada como un delito de lesa humanidad y exigir la sanción de una Convención de ONU sobre Desaparición Forzada de Personas, que permitiera el uso de la jurisdicción universal para investigar, juzgar y sancionar a los responsables de las desapariciones forzadas.

Este trabajo aproximativo pone en evidencia que aún resta mucho por investigar sobre el problema de las redes regionales transnacionales de defensa de los derechos humanos durante el último ciclo de dictaduras de Seguridad Nacional. En el caso de la FEDEFAM es necesario avanzar en indagaciones sobre cómo fue la interacción, recepción y aplicación de los lineamientos de esta organización regional en cada contexto nacional.

\section{Bibliografía}

Alonso, L. (2008). El surgimiento del movimiento argentino por los derechos humanos en perspectiva comparada. Páginas № 1, año

Alonso, L. (2010). Defensa de los Derechos Humanos frente a las dictaduras regresivas: los casos de Argentina y Uruguay en perspectiva comparada. Studia histórica. Historia contemporánea, núm. 28, 23-50.

Ayala, M. (2014). La formación de comités y redes de lucha contra la dictadura militar de los exiliados argentinos en Venezuela: interacciones locales, regionales y 


\section{Mario Ayala}

transnacionales (1976-1981). e-L@tina, Revista de Estudios Latinoamericanos, Vol. 12, núm. 46.

Ayala, M. (2017) Exiliados argentinos en Venezuela (1974-1983) (Tesis de Doctorado inédita). Facultad de Filosofía y Letras de la Universidad de Buenos Aires, Buenos Aires.

Badan Ribeiro, M.C. (2016) Exílio político brasileiro e circulação revolucionária internacional: um olhar para a Rede Solidariedade. Kamchatka. Revista de análisis cultural, $\mathrm{N}^{\circ}$ 8, Diciembre.

Catoggio, M.S. (2016). Política contra el Estado autoritario, religión y derechos humanos. La impronta regional de un activismo trasnacional. Papeles de Trabajo, vol. 10.

Catoggio, M.S. (2014). La trama religiosa de las redes humanitarias y el activismo transnacional en las dictaduras del Cono Sur de América Latina. En: Jensen, S. \& Lastra, S. (edits.). Exilios: militancia y represión. Nuevas fuentes, nuevos abordajes de los destierros de la Argentina en los años setenta. La Plata: EDULP.

Catoggio, M.S. (2012). Puentes latinoamericanos en el exilio en Francia: las redes trasnacionales del mundo religioso. Ponencia en Jornadas de Trabajo sobre Exilios Políticos del Cono Sur en el siglo XX, Universidad Nacional de La Plata, La Plata, Argentina.

Jelin, E. (2010). ¿Víctimas, familiares o ciudadano/ as? Las luchas por la legitimidad de la palabra. En Crenzel Emilio (coord.) Los desparecidos en la Argentina. Memorias, representaciones e ideas. 1983-2008. Buenos Aires: Biblos.

Dunkerley, J. (2003). Rebelión en las venas. La Paz: Plural.

Jensen, S. (2010). Los Exilados. La lucha por los derechos humanos durante la dictadura. Buenos Aires: Sudamericana.

Keck, M. y Sikkink, K. (1999). Las redes transnacionales de defensa en la política internacional y regionales. International Social Sciencie Journal, № 159.

Keck, M.; Sikkink, K. (2000). Activistas sin frontera: redes de defensa en política internacional. México: Siglo XXI.

Pettinà, V. (2018). Historia mínima de la guerra fría en América Latina. México: El Colegio de México.

Povedá Brito, Robert Alexander (2011) La teología de la liberación entre la Democracia y la Dictadura. Estudio de la labor de Fundalatin y el Padre Juan Vives Suriá en la defensa de la promoción de los derechos humanos y el desarrollo social 
Coordinaciones regionales humanitarias. Exiliados, religiosos y organismos de derechos humanos en la formación de la Federación Latinoamericana de Asociaciones de Familiares de Detenidos Desaparecidos (1979-1982)

entre 1978 y 1989, Tesis de Maestría en Historia de las Américas, Caracas, Universidad Católica Andrés Bello.

Rice, P. (2009). La Fédération Latino-Americaine des Organisations de Familles de Detenus Disparus (FEDEFAM) et le projet de Convention. En: Decaux, E.; De Frouville, 0. (dir.), La Convention pour la protection de toutes les personnes contre les disparitions forcées. [s.l.] coleccion Droit et Justice no 87, Bruylant.

Roniger, L. (2011a). Destierro y exilio en América Latina: Un campo de estudio transnacional e histórico en expansión. En: Revista Pacarina del Sur. Recuperado de: http://www.pacarinadelsur.com/home/abordajes-y-contiendas/318-destierro-yexilio-en-america-latina-un-campo-de-estudio-transnacional-e-historico-enexpansion

Roniger, L. (2011b). Reflexões sobre o exílio como tema de investigação: avanços teóricos e desafios. En: Quadrat, S. V. (Org.). Caminhos cruzados. História e memória dos exílios latino-americanos no século XX Rio de Janeiro: Editora de FVG.

Roniger, L. (2018) Historia mínima de los derechos humanos en América Latina. Ciudad de México: El Colegio de México.

Sagredo Mazuela, O. L. (2017). Redes transnacionales de defensa de los derechos humanos durante la primera fase de la dictadura chilena (1973-1976): formación y estrategias. Clepsidra. Revista Interdisciplinaria de Estudios sobre Memoria, 4 (7).

Sikkink, K. (2018). Razones para la esperanza. La legitimidad y efectividad de los derechos humanos de cara al futuro. Buenos Aires: Siglo XXI Editores.

Taylor, S.J., Bogdan, R. (1987) Introducción a los métodos cualitativos de investigación, Barcelona, Paidós.

Undurraga, J. (1983). La fuerza de los débiles. El modelo de las organizaciones de derechos humanos. Nueva Sociedad, Caracas, Nro. 64 Enero-Febrero.

Vázquez, L. (2012). Radios rebeldes. La revista del CCC [en línea], Enero / Agosto, $\mathrm{n}^{\circ}$ 14/15. Recuperado de: http://www.centrocultural.coop/revista/articulo/303/

\section{Documentos citados}

Equipo de Coordinación Fundalatin (1981b). Plan de Trabajo Julio-Diciembre de 1981. I Congreso Latinoamericano de Familiares de Desaparecidos. Caracas: FundaLatin, Mecanografiado, 6 de julio. 


\section{Mario Ayala}

FEDEFAM (1981믈. Documentos del I Congreso Latinoamericano de Familiares de Desaparecidos. Caracas: FEDEFAM. Archivo FEDEFAM.

FEDEFAM (1981b). Acta Final del II Congreso Latinoamericano de Familiares de Desaparecidos. Caracas: FundaLatin, 28 de noviembre. Mecanografiado, 10 pp.

FEDEFAM (1982b). Documentos del III Congreso Latinoamericano de Familiares de Desaparecidos. Caracas. Archivo FEDEFAM.

FundaLatin (1980). Convocatoria al Ier Congreso Latinoamericano de Familiares de Desaparecidos. Caracas, FundaLatin. Mecanografiado. Archivo personal de Alberto Ravara, Caracas.

FundaLatin (1981b). Presentación. Primer Congreso Latinoamericano de Familiares de Desaparecidos. Caracas-San José, FundaLatin, Enero. Mecanografiado. En FEDEFAM. Documentos del I Congreso Latinoamericano de Familiares de Desaparecidos. Caracas, p. 254-264. En Archivo FEDEFAM.

Jiménez Lozano, J. (1979), “La Conferencia Episcopal de Puebla”, El País, Madrid, 24 de enero.

Mealla, Luis (2013). Bolivia recuerda la masacre de 1979 con llamado de atención de la ONU. La Razón, La Paz, 01 de noviembre.

Oficina del Alto Comisionado de las Naciones Unidas para los Derechos Humanos (2019). Grupo de Trabajo sobre Desapariciones Forzadas o Involuntarias. Mandato. Recuperado de: https://www.ohchr.org/SP/Issues/Disappearances/Pages/DisappearancesIndex.a $\operatorname{spx}$

Departamento América Central y Caribe a Embajada Argentina en Guatemala, “Cable-Secreto-42622", Buenos Aires, 15 de septiembre de 1980. En AMREC, 94Coleccion Alfredo Forti.

Embajada Argentina en Costa Rica a Central Política de Informaciones, "CableSecreto-96675-676-677", Costa Rica, 17 de diciembre de 1980. En AMREC, 94Coleccion Alfredo Forti.

Revista 2001, Caracas, 01/02/1981.

El Día, México, 14/02/1981.

Equipo de Coordinación Fundalatin (1981a) Circular $\mathrm{N}^{\circ}$ 3. I Congreso Latinoamericano de Familiares de Desaparecidos. Caracas: FundaLatin, Mecanografiado, 6 de julio. Archivo personal de Alberto Ravara, Caracas. 
Coordinaciones regionales humanitarias. Exiliados, religiosos y organismos de derechos humanos en la formación de la Federación Latinoamericana de Asociaciones de Familiares de Detenidos Desaparecidos (1979-1982)

FundaLatin (1981a). Documento Base de FundaLatin en I Congreso de Familiares Desaparecidos de América Latina, Costa Rica, enero 1981. En FEDEFAM. Documentos del I Congreso Latinoamericano de Familiares de Desaparecidos, Caracas, p. 254-264, Archivo FEDEFAM.

FEDEFAM (1982a). Hasta Encontrarlos. Boletín Informativo, Año 1-Num. 1 - MayoJunio, Caracas.

Entrevistas citadas

Entrevistas con Patrick Rice, 12/12/2008 y 10/03/2010, Ciudad de Buenos Aires; Rice, 2009.

Entrevista con Viviana, 06/12/2008, City Bell, La Plata.

Correspondencia con Viviana, 21/07/2019, Córdoba.

Recibido: $25 / 01 / 2020$

Evaluado: $26 / 02 / 2020$

Versión Final: 15/03/2020 